\title{
SwissDRG: état des lieux critique d'un médecin de famille à l'attention des politiciens et des assureurs
}

Benedikt Horn

Correspondance:

Prof. Dr Benedikt Horn

Marktgasse 66

CH-3800 Interlaken

Tél. 0338221339

dr.horn@tcnet.ch

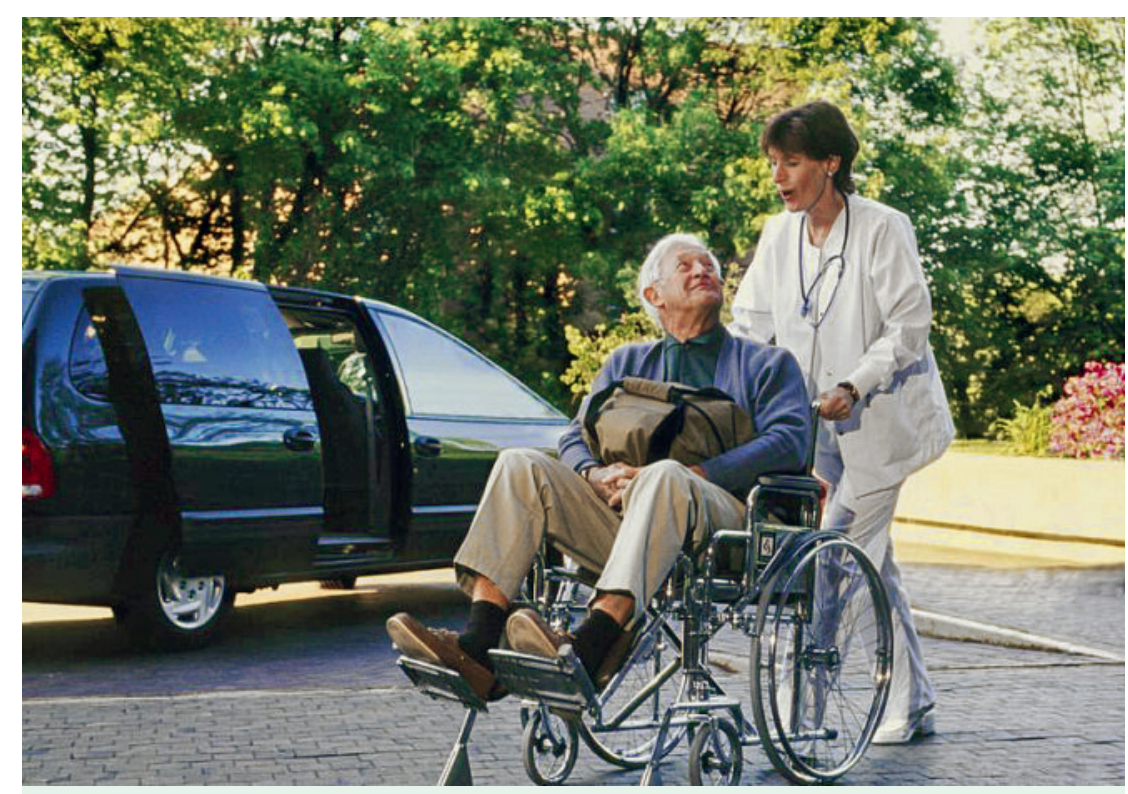

A la maison! Sont des sorties précoces un problème particulier du système DRG?

Dans le numéro 28/29 du Bulletin des médecins suisses, les confrères Hess et Strebel appelent à un moratorium des DRG en justifiant en détail cette démarche réfléchie. Dans le commentaire d'introduction de la Fédération des médecins suisses FMH, le confrère Cuénoud, responsable du ressort DRG du Comité central de la FMH, mentionne que les questions et les problèmes sont en grande partie justifiés et que la FMH partage la plupart des revendications. Néanmoins, la FMH ne soutient pas l'idée d'un moratoire: selon elle, il s'agit ici de l'application d'une loi qui a été édictée selon des règles démocratiques.

Afin d'épargner aux responsables de ne pas avoir entrevu les conséquences des DRG, plusieurs situations concrètes sont exposées d'une manière directe et réaliste. Il s'agit de l'interface hôpital-médecin de famille sensée être une transition, mais que l'introduction des DRG soumet à une épreuve de vérité.

\section{Quelques situations concrètes après I'introduction des DRG}

\section{Madame la Conseillère nationale!}

Nous sommes lundi à 16 h, la session commencera mardi à $9 \mathrm{~h}$ et vous êtes la présidente de fraction d'un grand parti. Avant-hier, votre père qui vit seul a fait une chute dans sa cuisine et s'est fracturé le col du fémur. «Pas de problème», a déclaré l'aimable médecin de jour du service d'urgence: opération sous anesthésie péridurale, intervention de routine, utilisation immédiate possible, cinq jours d'hospitalisation. Tout s'est bien passé. Votre mari, un avocat renommé, a avancé plusieurs rendez-vous afin de pouvoir veiller sur votre père pendant trois jours. Vous être membre du Conseil d'administration de ce grand hôpital, vous vous êtes engagée afin que le dépliant de l’hôpital mentionne que chez vous «le patient est au centre des préoccupations!», et vous étiez très sceptique face à l'introduction des forfaits par cas. Il vous a fallu de nombreux exposés et débats avec des experts pour vous convaincre que les DRG présentaient de nombreux avantages pour l'hôpital. Vous recevez maintenant un appel du service médical qui souhaite libérer votre père pour un besoin urgent de lits, cas d'urgence, etc. Tout va bien, le contrôle des plaies sera effectué par le médecin de famille et le service des soins à domicile, votre père a encore un drainage sus-pubien... Comment? Un drainage sus-pubien est un tuyau dans la vessie, pas de problème. Votre père est en train d'être chargé dans l'ambulance, il sera chez lui dans 20 minutes, merci pour votre compréhension, don't worry. Le médecin de famille? Il a fermé son cabinet il y a deux ans. Le centre de santé avec ses 6 médecins a certes bonne réputation, mais il se trouve à 15 kilomètres. Le personnel des soins à domicile est très qualifié, mais il ne vient que deux fois par jour, maximum 45 minutes au total. Que faire? No problem!

\section{Monsieur le Conseiller des Etats!}

Demain, c'est le grand jour! Vous avez investi une semaine de vos vacances pour rédiger votre plaidoyer contre les interventions de notre armée à l'étranger. La nuit dernière, votre épouse et très précieuse collaboratrice depuis vingt ans, a rédigé la version finale du manuscrit, écriture 16 points, interligne moyen, lisible sans lunette gênante. Au petit matin, elle s'est plainte de brûlures d'estomac rayonnantes dans les bras; pâle et très angoissée, son front était perlé de sueur. Immédiatement sur les lieux, le médecin de famille pense à un infarctus. Tranfert au centre hospitalier, cathéter cardiaque, ballon, deux stents... Son état est excellent et elle pourra rentrer chez elle demain à 
$7 \mathrm{~h}$ du matin. Votre fille est en vacances en NouvelleZélande et votre fils travaille aux Etats-Unis pour le Crédit Suisse. Et maintenant? il faudra lui injecter un médicament contre la thrombose jusqu'à ce qu'il ait recouvert son entière mobilité. Pas de problème, déclare le médecin du service au

\section{Vous recevez un appel du service médical qui souhaite libérer votre père pour un besoin urgent de lits}

\section{Madame la Conseillère des Etats!}

En tant que présidente de la Commission des finances, de nombreuses tâches vous attendent cet après-midi et votre présence est plus que jamais indispensable. Hier soir, votre fille unique a mis au monde un vaillant garçon, tout s'est bien passé, à part une forte hémorragie ultérieure, cela arrive. Le père de l'enfant accomplit son service militaire au Tessin et, en tant que commandant, sa présence est indispensable. C'est l'effervescence à la clinique, votre fille sera libérée dans une heure, elle est encore pâle, mais il faut un peu de patience. Visite à domicile de la sagefemme? Non, ce n'est pas prévu, le médecin de famille et les soins à domicile vont s'en occuper. Le médecin de famille? Votre fille n'a pas encore de médecin de famille, elle a toujours été en bonne santé, son médecin «en cas d'urgence» est sa gynécologue de l'hôpital situé à 15 kilomètres, mais elle ne fait pas de visites à domicile. Et alors? Où est le problème? téléphone, le médecin de famille en sera informé. Amené en ambulance, votre père est heureux de se retrouver chez lui. Son taux de glycémie n'a pas été aussi élevé depuis des années, sa tension non plus. L'urine qui s'écoule dans le cathéter de la vessie contient du sang. No problem...

\section{Monsieur le directeur!}

Vous êtes CEO d'une importante caisse-maladie. Il y a quelques jours, votre mère âgée qui vit seule a été ramenée chez elle en ambulance. Elle a été hospitalisée trois jours suite à un petit accident vasculaire cérébral. Les examens effectués au «Stroke Unit» se sont bien déroulés, le pronostic est bon. Votre mère est assez confuse, ne veut rien manger ni boire, elle ne peut pas se rendre seule aux toilettes. Tout va s'arranger, déclare le médecin du service. Vous tenez maintenant entre vos mains la facture du transport ambulancier qui correspond pratiquement au montant de la rente

\section{Les organes responsables doivent être franchement et clairement informés de ce qui attend nos patients et nous tous}

\section{Monsieur le Conseiller national!}

Vous êtes président de la Commission de la santé, vous vous êtes beaucoup intéressé aux forfaits par cas et êtes désormais convaincu qu'ils représentent une véritable chance de maittriser les coûts de la santé. Après-demain, un débat animé aura lieu sur la prévention, votre parti et le Ministre de la santé comptent sur vous! Votre père âgé, avec lequel vous vivez seul - loin de toute civilisation - dans une ferme, a dû être hospitalisé d'urgence justement hier. Jaunisse, apparemment un calcul de la vésicule biliaire. Intervention d'urgence, tout s'est bien passé malgré une tension élevée et du diabète. Depuis des années, vous effectuez deux injections d'insuline par jour à votre père et vous lui mesurez le taux de sucre plusieurs fois par jour. Les soins à domicile interviennent une fois par semaine et vous le conduisez tous les trois mois chez le médecin de famille. Le principal problème de votre père, ce sont ses yeux: «à cause du diabète»... Sinon, il va bien et pourra rentrer chez lui demain matin, il a encore un cathéter de la vessie et
AVS mensuelle. Le transport en hélicoptère vers le centre hospitalier est à la charge de l'hôpital. D'où l'hôpital tient-il cet argent? No problem. En résumé: les coûts ne sont pas économisés, ils sont au mieux déplacés, par exemple vers le secteur des transports.

\section{Bilan}

L'introduction des SwissDRG ou forfaits par cas ne semble plus guère être applicable. Les organes responsables doivent être franchement et clairement informés de ce qui attend nos patients et nous tous, et en particulier vous personnellement. Comme on le sait, le diable se cache dans les détails. Les patients sont désavantagés. No problem?

Un moratoire laisse le temps de résoudre les points critiques au lieu de créer de tels problèmes!

\section{Référence}

1 Hess C, Strebel U. DRG - Aufruf zu einem Moratorium. Bull Méd Suisses. 2010;91(28/29):1105-6. 


\section{Commentaire de Pierre-François Cuénoud}

Cher Confrère,

Qui d'entre nous n'a-t'il pas, dans sa carrière professionnelle, souhaité qu'un responsable politique ou I'un de ses proches soit touché dans sa santé, pour enfin pouvoir saisir concrètement les vrais enjeux du système sanitaire? Adepte de ce principe, votre propos est rafraîchissant. Cependant, le divertissement que procure sa lecture ne parvient pas à masquer l'impression d'être témoin d'un mauvais film. Quelles expériences amères avez-vous faites pour accorder un tel potentiel d'idiotie aux médecins hospitaliers? Vous semblez avoir une piètre idée du sens des responsabilités qui anime les médecins-cadres de nos établissements de soins aigus.

Sur le fond, vos exemples se concentrent sur le problème des sorties précoces. Le raccourcissement des séjours avec transfert d'activités au profit du secteur ambulatoire est une évolution que l'on constate dans tous les pays depuis une quinzaine d'années, indépendamment du mode de financement hospitalier. La recherche concomitante allemande et la seule étude comparative déjà effectuée en Suisse n'ont pas montré d'accélération significative de ce phénomène après l'introduction de forfaits par cas selon DRG. Ceci ne diminue cependant en rien la nécessité de procéder à un monitorage systématique des flux de patients au cours des prochaines années, dans le cadre d'une recherche concomitante crédible.

L'interface entre médecins hospitaliers et collègues de premier recours fonctionne et il n'y a pas de raison de penser que ces liens se dissolvent de manière abrupte lors de l'introduction de SwissDRG.
Les structures intermédiaires, destinées à assumer la phase de transition entre soins aigus et retour à domicile, se développent, souvent par reconversion de certains établissements hospitaliers aigus surnuméraires (les parents de vos décideurs ont beaucoup de malchance de résider dans une région dépourvue de tels établissements!). Le passage à des forfaits par cas selon DRG peut aussi être l'occasion de réfléchir à la création de centres de compétences plutôt qu'au maintien de petites structures hospitalières peu différenciées et dispersées. Les autorités cantonales, responsables de la santé de leur population, ne sont pas inactives et réalisent toute une série de projets. En particulier, elles sont nombreuses à adapter également leur législation en vue de l'échéance de 2012. Le monde actuel de la santé n'est plus cloisonné avec un grand ordonnateur médical gérant les affaires du haut de sa pyramide, même si cela éveille la nostalgie des plus anciens d'entre nous. Le trait manifestement exagéré de votre fresque sied mal à la réalité. Pourquoi de tels événements ne se sont-ils pas systématiquement produits dans les cantons qui appliquent les DRG depuis plusieurs années? Pourquoi les enquêtes régulières de satisfaction des patients n'y donnent-elles pas de plus mauvais résultats? Un contact avec les collègues actifs dans les hôpitaux aigus de ces régions vous montrera que l'éthique de notre profession n'y est pas plus bafouée qu'ailleurs.

Dr Pierre-François Cuénoud,

Membre du Comité Central de la FMH,

Responsable du domaine SwissDRG 